\title{
Face-degree bounds for planar critical graphs
}

\author{
Ligang Jin * Yingli Kang Eckhard Steffen \\ Institute of Mathematics and Paderborn Center for Advanced Studies \\ Paderborn University \\ Paderborn, Germany \\ \{ligang,yingli\}@mail.upb.de, es@upb.de
}

Submitted: Jan 26, 2016; Accepted: Jul 15, 2016; Published: Aug 5, 2016

\begin{abstract}
The only remaining case of a well known conjecture of Vizing states that there is no planar graph with maximum degree 6 and edge chromatic number 7 . We introduce parameters for planar graphs, based on the degrees of the faces, and study the question whether there are upper bounds for these parameters for planar edge-chromatic critical graphs. Our results provide upper bounds on these parameters for smallest counterexamples to Vizing's conjecture, thus providing a partial characterization of such graphs, if they exist.

For $k \leqslant 5$ the results give insights into the structure of planar edge-chromatic critical graphs.
\end{abstract}

Keywords: Vizing's planar graph conjecture; planar graphs; critical graphs; edge colorings

\section{Introduction}

We consider finite simple graphs $\mathrm{G}$ with vertex set $V(G)$ and edge set $E(G)$. The vertexdegree of $v \in V(G)$ is denoted by $d_{G}(v)$, and $\Delta(G)$ denotes the maximum vertex-degree of $G$. If it is clear from the context, then $\Delta$ is frequently used. The edge-chromaticnumber of $G$ is denoted by $\chi^{\prime}(G)$. Vizing [8] proved that $\chi^{\prime}(G) \in\{\Delta(G), \Delta(G)+1\}$. If $\chi^{\prime}(G)=\Delta(G)$, then $G$ is a class 1 graph, otherwise it is a class 2 graph. A class 2 graph $G$ is critical, if $\chi^{\prime}(H)<\chi^{\prime}(G)$ for every proper subgraph $H$ of $G$. Critical graphs with maximum vertex-degree $\Delta$ are also called $\Delta$-critical. It is easy to see that critical graphs are 2-connected. A graph $G$ is overfull if $|V(G)|$ is odd and $|E(G)| \geqslant \Delta(G)\left\lfloor\frac{1}{2}|V(G)|\right\rfloor+1$. Clearly, every overfull graph is class 2. A graph is planar if it can be embedded into the Euclidean plane. A plane graph $(G, \Sigma)$ is a planar graph $G$ together with an embedding

*supported by Deutsche Forschungsgemeinschaft (DFG) grant STE 792/2-1. 
$\Sigma$ of $G$ into the Euclidean plane. That is, $(G, \Sigma)$ is a particular drawing of $G$ in the Euclidean plane.

In 1964, Vizing [8] showed for each $k \in\{2,3,4,5\}$ that there is a planar class 2 graph $G$ with $\Delta(G)=k$. He proved that every planar graph with maximum vertex-degree at least 8 is a class 1 graph, and conjectured that every planar graph $H$ with $\Delta(H) \in\{6,7\}$ is a class 1 graph. Vizing's conjecture has been proved for planar graph with maximum vertex-degree 7 by Grünewald [3], Sanders and Zhao [6], and Zhang [13] independently.

Zhou [14] proved for each $k \in\{3,4,5\}$ that if $G$ is a planar graph with $\Delta(G)=6$ and $G$ does not contain a circuit of length $k$, then $G$ is a class 1 graph. Vizing's conjecture is confirmed for some other classes of planar graphs which do not contain some specific (chordal) circuits $[1,10,11]$.
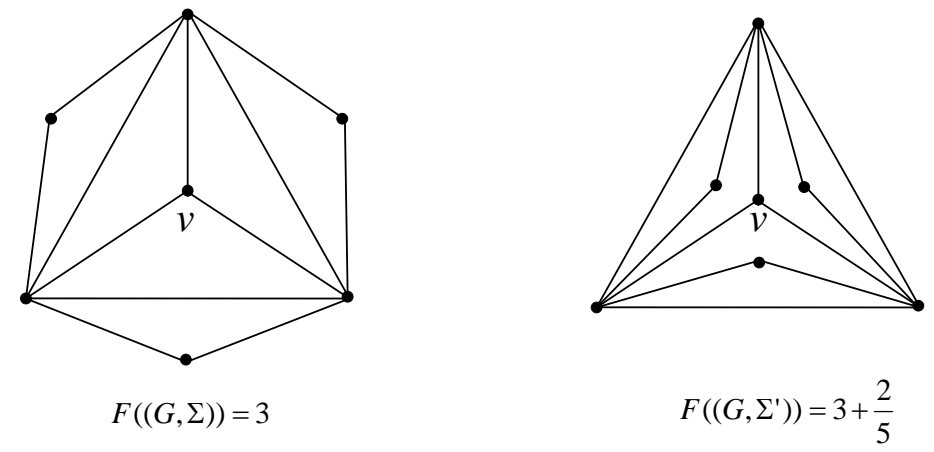

Figure 1: Graph $G$ has two embeddings $\Sigma, \Sigma^{\prime}$ such that $F((G, \Sigma)) \neq F\left(\left(G, \Sigma^{\prime}\right)\right)$.

Let $G$ be a 2-connected planar graph, $\Sigma$ be an embedding of $G$ in the Euclidean plane and $F(G)$ be the set of faces of $(G, \Sigma)$. The degree $d_{(G, \Sigma)}(f)$ of a face $f$ is the length of its facial circuit. If there is no harm of confusion we also write $d_{G}(f)$ instead of $d_{(G, \Sigma)}(f)$. Let $\bar{F}(G)=\frac{1}{|F(G)|} \sum_{f \in F(G)} d_{G}(f)$ be the average face-degree of $G$. Euler's formula $|V(G)|-|E(G)|+|F(G)|=2$ implies that $\bar{F}(G)=\frac{2|E(G)|}{|E(G)|-|V(G)|+2}$.

Let $v \in V(G)$. If $d_{G}(v)=k$, then $v$ is incident to $k$ pairwise different faces $f_{1}, \ldots, f_{k}$. Let $F_{(G, \Sigma)}(v)=\frac{1}{k}\left(d_{(G, \Sigma)}\left(f_{1}\right)+\cdots+d_{(G, \Sigma)}\left(f_{k}\right)\right)$ and $F((G, \Sigma))=\min \left\{F_{(G, \Sigma)}(v): v \in\right.$ $V(G)$ \}. Clearly, $F((G, \Sigma)) \geqslant 3$ since every face has length at least 3. As Figure 1 shows, $F((G, \Sigma))$ depends on the embedding $\Sigma$. The local average face-degree of a 2-connected planar graph $G$ is

$$
F^{*}(G)=\max \{F((G, \Sigma)):(G, \Sigma) \text { is a plane graph }\} .
$$

This parameter is independent from the embeddings of $G$, and $F^{*}(G) \geqslant 3$ for all planar graphs. Let $k$ be a positive integer. Let $\bar{b}_{k}=\sup \{\bar{F}(G): G$ is a $k$-critical planar graph $\}$ and $b_{k}^{*}=\sup \left\{F^{*}(G): G\right.$ is a $k$-critical planar graph $\}$. We call $\bar{b}_{k}$ the the average facedegree bound, and $b_{k}^{*}$ the local average face-degree bound for $k$-critical planar graphs. If $k=1$ or $k \geqslant 7$, then every planar graph with maximum vertex-degree $k$ is a class 1 graph and therefore, $\{\bar{F}(G): G$ is a $k$-critical planar graph $\}=\left\{F^{*}(G): G\right.$ is a $k$-critical 
planar graph $\}=\varnothing$. Hence, $\bar{b}_{k}$ and $b_{k}^{*}$ do not exist in these cases. Therefore, we focus on $k \in\{2,3,4,5,6\}$ in this paper. The main results are the following two theorems.

Theorem 1. Let $k \geqslant 2$ be an integer.

- If $k=2$, then $\bar{b}_{k}=\infty$.

- If $k=3$, then $6 \leqslant \bar{b}_{k} \leqslant 8$.

- If $k=4$, then $4 \leqslant \bar{b}_{k} \leqslant 4+\frac{4}{5}$

- If $k=5$, then $3+\frac{1}{3} \leqslant \bar{b}_{k} \leqslant 3+\frac{3}{4}$.

- If $k=6$ and $\bar{b}_{k}$ exists, then $\bar{b}_{k} \leqslant 3+\frac{1}{3}$.

Theorem 2. Let $k \geqslant 2$ be an integer.

- If $k \in\{2,3,4\}$, then $b_{k}^{*}=\infty$.

- If $k=5$, then $3+\frac{1}{5} \leqslant b_{k}^{*} \leqslant 7+\frac{1}{2}$.

- If $k=6$ and $b_{k}^{*}$ exists, then $b_{k}^{*} \leqslant 3+\frac{2}{5}$.

Vizing [9] proved that a class 2 graph contains $k$-critical subgraph for each $k \in$ $\{2, \ldots, \Delta\}$. Hence a smallest counterexample to Vizing's conjecture is critical and thus, our results for $k=6$ partially characterize smallest counterexamples to this conjecture. For $k \leqslant 5$, they provide insight into the structure of planar critical graphs. Seymour's exact conjecture [7] says that every critical planar graph is overfull. If this conjecture is true for $k \in\{3,4,5\}$, then $\bar{b}_{k}$ is equal to the lower bound given in Theorem 1 .

It is not clear whether $\bar{b}_{k}$ and $b_{k}^{*}$ or $\bar{F}(G)$ and $F^{*}(G)$ are related to each other, respectively. Furthermore, the precise values of $\bar{b}_{k}$ and $b_{k}^{*}$ are also unknown.

The next section states some properties of critical and of planar graphs. These results are used for the proofs of Theorems 1 and 2 which are given in Section 3.

\section{Preliminaries}

Let $G$ be a 2 -connected graph. A vertex $v$ is called a $k$-vertex, or a $k^{+}$-vertex, or a $k^{-}$vertex if $d_{G}(v)=k$, or $d_{G}(v) \geqslant k$, or $d_{G}(v) \leqslant k$, respectively. Let $N(v)$ be the set of vertices which are adjacent to $v$, and $N(S)=\bigcup_{v \in S} N(v)$ for a set $S \subseteq V(G)$. We write $N(v)$ and $N(u, v)$ short for $N(\{v\})$ and $N(\{u, v\})$, respectively.

Let $(G, \Sigma)$ be a plane graph. A face $f$ is called $k$-face, or a $k^{+}$-face, or a $k^{-}$-face, if $d_{(G, \Sigma)}(f)=k$, or $d_{(G, \Sigma)}(f) \geqslant k$, or $d_{(G, \Sigma)}(f) \leqslant k$, respectively. We will use the following well-known results on critical graphs.

Lemma 3. Let $G$ be a critical graph and $e \in E(G)$. If $e=x y$, then $d_{G}(x) \geqslant 2$, and $d_{G}(x)+d_{G}(y) \geqslant \Delta(G)+2$. 
Lemma 4 (Vizing's Adjacency Lemma [8]). Let $G$ be a critical graph. If $e=x y \in E(G)$, then at least $\Delta(G)-d_{G}(y)+1$ vertices in $N(x) \backslash\{y\}$ have degree $\Delta(G)$.

Lemma 5 ([13]). Let $G$ be a critical graph and $x y \in E(G)$. If $d(x)+d(y)=\Delta(G)+2$, then

1. every vertex of $N(x, y) \backslash\{x, y\}$ is a $\Delta(G)$-vertex,

2. every vertex in $N(N(x, y)) \backslash\{x, y\}$ has degree at least $\Delta(G)-1$,

3. if $d(x)<\Delta(G)$ and $d(y)<\Delta(G)$, then every vertex in $N(N(x, y)) \backslash\{x, y\}$ has degree $\Delta(G)$.

Lemma 6 ([6]). No critical graph has pairwise distinct vertices $x, y, z$, such that $x$ is adjacent to $y$ and $z, d(z)<2 \Delta(G)-d(x)-d(y)+2$, and $x z$ is in at least $d(x)+d(y)-$ $\Delta(G)-2$ triangles not containing $y$.

We will use the following results on lower bounds for the number of edges in critical graphs.

Theorem 7 ([4]). If $G$ is a 3-critical graph, then $|E(G)| \geqslant \frac{4}{3}|V(G)|$.

Theorem 8 ([12]). Let $G$ be a k-critical graph. If $k=4$, then $\mid E\left(G\left|\geqslant \frac{12}{7}\right| V(G) \mid\right.$, and if $k=5$, then $|E(G)| \geqslant \frac{15}{7}|V(G)|$.

Theorem 9 ([5]). If $G$ is a 6-critical graph, then $|E(G)| \geqslant \frac{1}{2}(5|V(G)|+3)$.

Lemma 10. Let $t$ be a positive integer and $\epsilon>0$.

1. For $k \in\{2,3,4\}$ there is a k-critical planar graph $G$ and $F^{*}(G)>t$.

2. There is a 2-critical planar graph $G$ with $\bar{F}(G)>t$.

3. There is a 3-critical planar graph $G$ such that $6-\epsilon<\bar{F}(G)<6$.

4. There is a 4-critical planar graph $G$ such that $4-\epsilon<\bar{F}(G)<4$.

5. There is a 5-critical planar graph $G$, such that $3+\frac{1}{3}-\epsilon<\bar{F}(G)<3+\frac{1}{3}$ and $F^{*}(G) \geqslant 3+\frac{1}{5}$.

Proof. The odd circuits are the only 2-critical graphs. Hence, the second statement and the first statement for $k=2$ are proved. Let $X$ and $Y$ be two circuits of length $n \geqslant 3$, with $V(X)=\left\{x_{i}: 0 \leqslant i \leqslant n-1\right\}, V(Y)=\left\{y_{i}: 0 \leqslant i \leqslant n-1\right\}$ and edges $x_{i} x_{i+1}$ and $y_{i} y_{i+1}$, where the indices are added modulo $n$. Consider an embedding, where $Y$ is inside $X$. Add edges $x_{i} y_{i}$ to obtain a planar cubic graph $G$ with $F^{*}(G)=\frac{1}{3}(n+8)$. Add edges $x_{i} y_{i+1}$ in $G$ to obtain a 4-regular planar graph $H$ with $F^{*}(H)=\frac{1}{4}(n+9)$. Subdividing one edge in $G$ and one in $H$ yields a critical planar graph $G_{n}$ with $\Delta\left(G_{n}\right)=3$, and a critical planar graph $H_{n}$ with $\Delta\left(H_{n}\right)=4$. If $n \geqslant 4 t$, then $F^{*}\left(G_{n}\right)>t$ and $F^{*}\left(H_{n}\right)>t$. The proof that $G_{n}$ and $H_{n}$ are critical will be given in the last paragraph. 


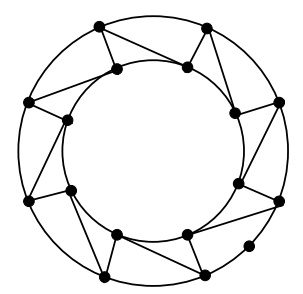

$k=4$

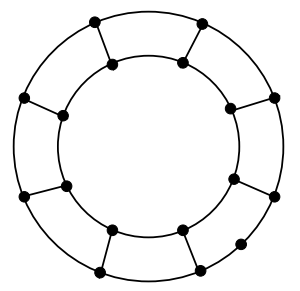

$k=3$

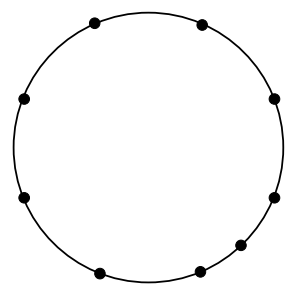

$k=2$

Figure 2: Examples for $k \in\{2,3,4\}$

Since $\left|F\left(G_{n}\right)\right|=n+2$, and $\sum_{f \in F\left(G_{n}\right)} d_{G_{n}}(f)=6 n+2$, it follows that $\bar{F}\left(G_{n}\right)=6-\frac{10}{n+2}$. Analogously, we have $\left|F\left(H_{n}\right)\right|=2 n+2$ and $\sum_{f \in F\left(H_{n}\right)} d_{H_{n}}(f)=8 n+2$ and therefore, $\bar{F}\left(H_{n}\right)=4-\frac{3}{n+1}$. Now, the statements for 3 -critical and 4-critical graphs follow. Examples of these graphs are given in Figure 2.

Let $m \geqslant 4$ be an integer. Let $C_{i}=\left[c_{i, 1} c_{i, 2} \cdots c_{i, 4}\right]$ be a circuit of length 4 for $i \in\{1, m\}$, and $C_{i}=\left[c_{i, 1} c_{i, 2} \cdots c_{i, 8}\right]$ be a circuit of length 8 for $i \in\{2, \ldots, m-1\}$. Consider an embedding, where $C_{i}$ is inside $C_{i+1}$ for $i \in\{1, \ldots, m-1\}$. Add edges $c_{1, j} c_{2,2 j-1}, c_{1, j} c_{2,2 j}$, $c_{1, j} c_{2,2 j+1}$ for $j \in\{1, \ldots, 4\}$, edges $c_{i, j} c_{i+1, j}$ for $i \in\{2, \ldots, m-2\}$ and $j \in\{1, \ldots, 8\}$, edges $c_{i, j} c_{i+1, j+1}$ for $i \in\{2, \ldots, m-2\}$ and $j \in\{2,4,6,8\}$, and edges $c_{m-1,2 j-2} c_{m, j}, c_{m-1,2 j-1} c_{m, j}$ and $c_{m-1,2 j} c_{m, j}$ for $j \in\{1, \ldots, 4\}$ to obtain a 5-regular planar graph $T$ (the indices are added modulo 8). Subdividing the edge $c_{m, 1} c_{m, 2}$ in $T$ yields a critical planar graph $T_{m}$ with $\Delta\left(T_{m}\right)=5$ (Figure 3 illustrates $\left.T_{6}\right)$.

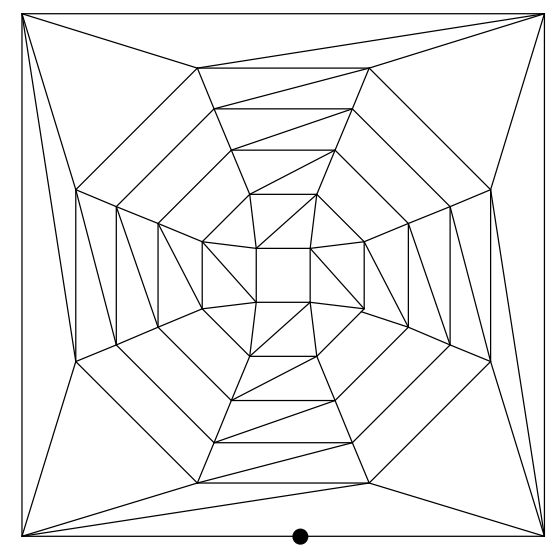

Figure 3: $\left(T_{6}, \Sigma_{6}\right)$

Since $\left|F\left(T_{m}\right)\right|=12 m-10$ and $\sum_{f \in F\left(T_{m}\right)} d_{T_{m}}(f)=40 m-38$, it follows that $\bar{F}\left(T_{m}\right)=$ $\frac{10}{3}-\frac{7}{18 m-15}$. Furthermore, for the embedding $\Sigma_{m}$ of $T_{m}$ as indicated in Figure 3 (for $m=6)$ we calculate that $F\left(\left(T_{m}, \Sigma_{m}\right)\right)=3+\frac{1}{5}$ and therefore, $F^{*}\left(T_{m}\right) \geqslant 3+\frac{1}{5}$.

It remains to prove that $G_{n}, H_{n}$ and $T_{m}$ are critical. For $G_{n}$ and $H_{n}$ we proceed by induction on $n$. It is easy to verify the truth for $3 \leqslant n \leqslant 6$. We proceed to induction 
step. We argue first on $G_{n}$. Let $u$ be the vertex of degree 2. Since $n \geqslant 7$, for any edge $e$ of $G_{n}$, there exists some $k$ such that no vertex of the circuit $C$ is incident with $e$ or adjacent to $u$, where $C=\left[x_{k+1} y_{k+1} y_{k+2} x_{k+2}\right]$. Reduce $G_{n}$ to $G_{n-2}$ by removing the edges $x_{k+1} y_{k+1}$ and $x_{k+2} y_{k+2}$ and suppressing their ends. Let $G^{\prime}$ be the resulting graph and $e^{\prime}$ be the resulting edge from $e$. By the induction hypothesis, $G^{\prime}$ is critical. Hence, $G^{\prime}-e^{\prime}$ has a 3-edge-coloring, say $\phi$. Assign $\phi\left(x_{k} x_{k+3}\right)$ to $x_{k} x_{k+1}$ and $x_{k+2} x_{k+3}$, and $\phi\left(y_{k} y_{k+3}\right)$ to $y_{k} y_{k+1}$ and $y_{k+2} y_{k+3}$, and consequently, the edges of $C$ can be properly colored. Now a 3-edge-coloring of $G_{n}-e$ is completed and so, $G_{n}-e$ is class 1 . Moreover, since $G_{n}$ is overfull, this graph is class 2. Therefore, $G_{n}$ is critical. The argument on $H_{n}$ is analogous.

For any $T_{m}$, recall that $T$ is the graph obtained from $T_{m}$ by suppressing the bivalent vertex. Consider $T$. Since each circuit $C_{i}$ has even length, their edges can be decomposed into two perfect matchings $M_{1}$ and $M_{2}$, so that $M_{1}$ contains $c_{i, 1} c_{i, 2}$ for $i \in\{1, m\}$ and $c_{i, 2} c_{i, 3}$ for $2 \leqslant i \leqslant m-1$. Let $M_{3}=\left\{c_{1, j} c_{2,2 j+1}: 1 \leqslant j \leqslant 4\right\} \cup\left\{c_{i, 2 j} c_{i+1,2 j+1}: 2 \leqslant i \leqslant\right.$ $m-2,1 \leqslant j \leqslant 4\} \cup\left\{c_{m-1,2 j-2} c_{m, j}: 1 \leqslant j \leqslant 4\right\}$. Clearly, $M_{3}$ is a perfect matching disjoint with $M_{1}$ and $M_{2}$. We can see that $E(G) \backslash\left(M_{1} \cup M_{2} \cup M_{3}\right)$ induces even circuits and hence, their edges can be decomposed into two perfect matchings $M_{4}$ and $M_{5}$, so that $M_{4}$ contains $c_{1, j} c_{2,2 j}$ for $1 \leqslant j \leqslant 4$. Clearly, $M_{1}, \ldots, M_{5}$ constitute a decomposition of $E(T)$.

Let $e_{i}=c_{m, i} c_{m, i+1}$ for $1 \leqslant i \leqslant 4$. Let $M_{2}^{\prime}=M_{2} \cup\left\{e_{1}, e_{3}\right\} \backslash\left\{e_{2}, e_{4}\right\}$. Define $A_{1}=$ $M_{1} \cup M_{3}, A_{2}=M_{2}^{\prime} \cup M_{4}, A_{3}=M_{2}^{\prime} \cup M_{5}$.

Let $h_{m}$ be an edge of $T_{m}$. Since $T_{m}$ is overfull, to prove that $T_{m}$ is critical, it suffices to show that $T_{m}-h_{m}$ is a 5-edge-colorable.

Let $h$ be the edge of $T$ that corresponds to $h_{m}$. We can see that $A_{1} \cup A_{2} \cup A_{3}=E(T) \backslash$ $\left\{e_{2}, e_{4}\right\}$ and $e_{1} \in A_{1} \cap A_{2} \cap A_{3}$. Hence, if $h \notin\left\{e_{2}, e_{4}\right\}$ then there exists $A \in\left\{A_{1}, A_{2}, A_{3}\right\}$ such that $e_{1}, h \in A$. Note that $e_{1}$ is the edge subdivided to get $T_{m}$ from $T$, and that $A$ induces a circuit of $T$. It follows that this circuit corresponds to a path $P$ of $T_{m}-h_{m}$. Moreover, note that the edges of $T-A$ can be decomposed into 3 perfect matchings, and thus the same to the edges of $T_{m}-h_{m}-E(P)$. Therefore, $T_{m}-h_{m}$ is 5 -edge-colorable.

If $h \in\left\{e_{2}, e_{4}\right\}$ then $C_{m}$ corresponds to a path of $T_{m}-h_{m}$. Note that $E\left(C_{m}\right) \subseteq M_{1} \cup M_{2}$ and that $M_{1}, \ldots, M_{5}$ constitute a decomposition of $E(T)$. Similarly, we can argue that $T_{m}-h_{m}$ is 5-edge-colorable in this case.

The following lemma is implied by Euler's formula directly.

Lemma 11. If $G$ is a planar graph, then $|E(G)|=\frac{\bar{F}(G)}{\bar{F}(G)-2}(|V(G)|-2)$.

\section{Proofs}

\subsection{Theorem 1}

The statement for $k=2$ and the lower bounds for $\bar{b}_{k}$ if $k \in\{3,4,5\}$ follow from Lemma 10. The other statements of Theorem 1 are implied by the following proposition.

Proposition 12. Let $G$ be a k-critical planar graph.

1. If $k=3$, then $\bar{F}(G)<8$. 
2. If $k=4$, then $\bar{F}(G)<4+\frac{4}{5}$.

3. If $k=5$, then $\bar{F}(G)<3+\frac{3}{4}$.

4. If $k=6$, then $\bar{F}(G)<3+\frac{1}{3}$.

Proof. Let $k=3$ and suppose to the contrary that $\bar{F}(G) \geqslant 8$. With Lemma 11 and Theorem 7 we deduce $\frac{4}{3}|V(G)| \leqslant|E(G)| \leqslant \frac{4}{3}(|V(G)|-2)$, a contradiction.

The other statements follow analogously from Lemma 11 and Theorem $8(k \in\{4,5\})$ and Theorem $9(k=6)$.

\subsection{Theorem 2}

The statement for $k \in\{2,3,4\}$ and the lower bound for $b_{5}^{*}$ follow from Lemma 10 . It remains to prove the upper bounds for $b_{5}^{*}$ and $b_{6}^{*}$. The result for $b_{5}^{*}$ is implied by the following theorem.

Theorem 13. If $G$ is a planar 5 -critical graph, then $F^{*}(G) \leqslant 7+\frac{1}{2}$.

Proof. Suppose to the contrary that $F^{*}(G)=r>7+\frac{1}{2}$. Let $\Sigma$ be an embedding of $G$ into the Euclidean plane such that $F^{*}(G)=F((G, \Sigma))$. Let $V=V(G), E=E(G)$, and $F$ be the set of faces of $(G, \Sigma)$. We proceed by a discharging argument in $G$ and eventually deduce a contradiction. Define the initial charge $\operatorname{ch}$ in $G$ as $\operatorname{ch}(x)=d_{G}(x)-4$ for $x \in V \cup F$. Euler's formula $|V|-|E|+|F|=2$ can be rewritten as:

$$
\sum_{x \in V \cup F} \operatorname{ch}(x)=\sum_{x \in V \cup F}\left(d_{G}(x)-4\right)=-8 .
$$

We define suitable discharging rules to change the initial charge function $c h$ to the final charge function $c h^{*}$ on $V \cup F$ such that $\sum_{x \in V \cup F} c h^{*}(x) \geqslant 0$ for all $x \in V \cup F$. Thus,

$$
-8=\sum_{x \in V \cup F} \operatorname{ch}(x)=\sum_{x \in V \cup F} c h^{*}(x) \geqslant 0,
$$

which is the desired contradiction.

Note that if a face $f$ sends charge $-\frac{1}{3}$ to a vertex $y$, then this can also be considered as $f$ receives charge $\frac{1}{3}$ from $y$. The discharging rules are defined as follows.

R1: Every $3^{+}$-face $f$ sends $\frac{d_{G}(f)-4}{d_{G}(f)}$ to each incident vertex.

R2: Let $y$ be a 5 -vertex of $G$.

R2.1: If $z$ is a 2-neighbor of $y$, then $y$ sends $\frac{2}{3}+\frac{2}{\lceil 2 r\rceil-3}$ to $z$.

R2.2: If $z$ is a 3-neighbor of $y$, then $y$ sends charge to $z$ as follows:

R2.2.1: if $z$ has a 4-neighbor, then $y$ sends $\frac{1}{3}+\frac{2}{\lceil 3 r\rceil-6}$ to $z$;

R2.2.2: if $z$ has no 4-neighbor, then $y$ sends $\frac{2}{9}+\frac{4}{3(\lceil 3 r\rceil-6)}$ to $z$.

R2.3: If $z$ is a 4-neighbor of $y$ and $z$ is adjacent to $n$-vertices $(2 \leqslant n \leqslant 4)$, then $y$ sends $\frac{4}{n(\lceil 4 r\rceil-9)}$ to $z$.

R2.4: If $y$ is adjacent to five $4^{+}$-vertices, then $y$ sends $\frac{1}{3}\left(\frac{4}{\lceil 5 r\rceil-12}+\frac{2}{\lceil 2 r\rceil-3}\right)$ to each 5 -neighbor which is adjacent to a 2 -vertex. 
Claim 14. If $u$ is a $k$-vertex, then $u$ receives at least $\frac{4-k}{3}-\frac{4}{\lceil r k\rceil-3 k+3}$ in total from its incident faces by $R 1$. In particular, if $u$ is incident with at most two triangles, then $u$ receives at least $\frac{1}{3}-\frac{4}{\lceil r k\rceil-4 k+6}$ in total from its incident faces.

Proof. Note that if $a$ and $b$ are integers and $2 \leqslant a \leqslant b$, then $\frac{1}{a-1}+\frac{1}{b+1} \geqslant \frac{1}{a}+\frac{1}{b}$.

Let $u$ be a $k$-vertex which is incident with faces $f_{1}, f_{2}, \cdots, f_{k}$. According to rule R1, $u$ totally receives charge $S=\sum_{i=1}^{k} \frac{d_{G}\left(f_{i}\right)-4}{d_{G}\left(f_{i}\right)}=k-4 \sum_{i=1}^{k} \frac{1}{d_{G}\left(f_{i}\right)}$ from its incident faces. The supposition $r \geqslant \frac{15}{2}$ implies that not all of $f_{1}, \ldots, f_{k}$ are triangles. It follows by $(\otimes)$ that $\sum_{i=1}^{k} \frac{1}{d_{G}\left(f_{i}\right)}$ reaches its maximum when all of $f_{1}, \ldots, f_{k}$ are triangles except one. Since $\sum_{i=1}^{k} d_{G}\left(f_{i}\right) \geqslant\lceil r k\rceil$, we have $S \geqslant k-4\left(\frac{1}{3}(k-1)+\frac{1}{\lceil r k\rceil-3(k-1)}\right)=\frac{4-k}{3}-\frac{4}{\lceil r k\rceil-3 k+3}$. In particular, if $u$ is incident with at most two triangles, then we have $S \geqslant k-4\left(\frac{2}{3}+\frac{1}{4}(k-\right.$ $\left.3)+\frac{1}{\lceil r k\rceil-6-4(k-3)}\right)=\frac{1}{3}-\frac{4}{\lceil r k\rceil-4 k+6}$.

Claim 15. The charge that a 5-vertex sends to a 4-neighbor by R2.3 is smaller than or equal to the charge that a 5-vertex sends to a 5-neighbor which is adjacent to a 2-vertex by R2.4, that is, $\frac{4}{n(\lceil 4 r\rceil-9)} \leqslant \frac{1}{3}\left(\frac{4}{\lceil 5 r\rceil-12}+\frac{2}{\lceil 2 r\rceil-3}\right)$.

Proof. Since $\frac{4}{n(\lceil 4 r\rceil-9)} \leqslant \frac{2}{\lceil 4 r\rceil-9} \leqslant \frac{2}{4 r-9}$ and $\frac{1}{3}\left(\frac{4}{5 r+1-12}+\frac{2}{2 r+1-3}\right) \leqslant \frac{1}{3}\left(\frac{4}{\lceil 5 r\rceil-12}+\frac{2}{\lceil 2 r\rceil-3}\right)$, we only need to prove that $\frac{2}{4 r-9} \leqslant \frac{1}{3}\left(\frac{4}{5 r+1-12}+\frac{2}{2 r+1-3}\right)$, which is equivalent to $2 r^{2}-15 r+23 \geqslant 0$ by simplification. Clearly, this inequality is true for every $r \geqslant 5+\frac{2}{5}$.

It remains to check the final charge for all $x \in V \cup F$.

Let $f \in F$, then $c h^{*}(f) \geqslant d_{G}(f)-4-d_{G}(f) \frac{d_{G}(f)-4}{d_{G}(f)}=0$ by R 1 .

Let $v \in V$. If $d_{G}(v)=2$, then $v$ receives at least $\frac{2}{3}-\frac{4}{\lceil 2 r\rceil-3}$ in total from its incident faces by Claim 14. By Lemma 3, $v$ has two 5-neighbors. Thus, $v$ receives $\frac{2}{3}+\frac{2}{\lceil 2 r\rceil-3}$ from each of them by R2.1. So we have $c h^{*}(v) \geqslant d_{G}(v)-4+\left(\frac{2}{3}-\frac{4}{\lceil 2 r\rceil-3}\right)+2\left(\frac{2}{3}+\frac{2}{\lceil 2 r\rceil-3}\right)=0$.

If $d_{G}(v)=3$, then $v$ receives at least $\frac{1}{3}-\frac{4}{\lceil 3 r\rceil-6}$ in total from its incident faces by Claim 14. By Lemmas 3 and $4, v$ has three $4^{+}$-neighbors, and two of them have degree 5 . If $v$ has a 4-neighbor, then by R2.2.1, $c h^{*}(v) \geqslant d_{G}(v)-4+\left(\frac{1}{3}-\frac{4}{\lceil 3 r\rceil-6}\right)+2\left(\frac{1}{3}+\frac{2}{\lceil 3 r\rceil-6}\right)=0$. Otherwise, by R2.2.2, $c h^{*}(v) \geqslant d_{G}(v)-4+\left(\frac{1}{3}-\frac{4}{\lceil 3 r\rceil-6}\right)+3\left(\frac{2}{9}+\frac{4}{3(\lceil 3 r\rceil-6)}\right)=0$.

If $d_{G}(v)=4$, then $v$ receives at least $-\frac{4}{[4 r]-9}$ in total from its incident faces by Claim 14. Say $v$ has precisely $n 5$-neighbors. By Lemma 3 , we have $2 \leqslant n \leqslant 4$. By R2.3, each of these 5-neighbors send $\frac{4}{n(\lceil 4 r\rceil-9)}$ to $v$. Therefore, $c h^{*}(v) \geqslant d_{G}(v)-4-\frac{4}{\lceil 4 r\rceil-9}+n \frac{4}{n(\lceil 4 r\rceil-9)}=0$.

If $d_{G}(v)=5$, then $v$ receives at least $-\frac{1}{3}-\frac{4}{\lceil 5 r\rceil-12}$ in total from its incident faces by Claim 14. First assume $v$ has a 2-neighbor, then by Lemma 5, $v$ has four 5-neighbors and at least three of them are adjacent to no $3^{-}$-vertex. Hence, by R2.1 and R2.4, $c h^{*}(v) \geqslant d_{G}(v)-4-\left(\frac{1}{3}+\frac{4}{\lceil 5 r\rceil-12}\right)-\left(\frac{2}{3}+\frac{2}{\lceil 2 r\rceil-3}\right)+3\left(\frac{1}{3}\left(\frac{4}{\lceil 5 r\rceil-12}+\frac{2}{\lceil 2 r\rceil-3}\right)\right)=0$.

Next assume that $v$ has a 3-neighbor $u$, then by Lemma $4, v$ has at least three 5neighbors. In this case, $v$ sends nothing to each 5 -neighbor. Let $w$ be the remaining neighbor of $v$. Then $d_{G}(w) \in\{3,4,5\}$.

If $d_{G}(w)=3$, then $u w \notin E(G)$ by Lemma 3. Furthermore, Lemma 6 implies that neither $v w$ nor $u v$ is contained in a triangle. It follows that $v$ is incident with at most two 
triangles. Thus, by Claim 14, $v$ receives a charge of at least $\frac{1}{3}-\frac{4}{\lceil 5 r]-14}$ in total from its incident faces. Moreover, both $u$ and $w$ have no $4^{-}$-neighbors. Suppose to the contrary that $t$ is a $4^{-}$-neighbor of $u$ (analogously of $w$ ). By Lemma 3, we have $d_{G}(t)=4$. By applying Lemma 5 to $u t$, we have $d_{G}(w) \geqslant 4$, a contradiction. Hence, $v$ sends $\frac{2}{9}+\frac{4}{3(\lceil 3 r\rceil-6)}$ to each of $u$ and $w$ by rule $R 2.2 .2$, yielding $c h^{*}(v) \geqslant d_{G}(v)-4+\left(\frac{1}{3}-\frac{4}{\lceil 5 r\rceil-14}\right)-2\left(\frac{2}{9}+\right.$ $\left.\frac{4}{3(\lceil 3 r\rceil-6)}\right)=\frac{8}{9}-\frac{4}{\lceil 5 r\rceil-14}-\frac{8}{3(\lceil 3 r\rceil-6)}$.

If $d_{G}(w)=4$, and if $u$ is adjacent to $w$, then by Lemma 5, $w$ has three 5-neighbors. Hence, by R2.2 and R2.3, $c h^{*}(v) \geqslant d_{G}(v)-4-\left(\frac{1}{3}+\frac{4}{\lceil 5 r\rceil-12}\right)-\left(\frac{1}{3}+\frac{2}{\lceil 3 r\rceil-6}\right)-\frac{4}{3(\lceil 4 r\rceil-9)}=$ $\frac{1}{3}-\frac{2}{\lceil 3 r\rceil-6}-\frac{4}{3(\lceil 4 r\rceil-9)}-\frac{4}{\lceil 5 r\rceil-12}$. If $u$ is not adjacent to $w$, then for any neighbor $t$ of $u$, we have $d_{G}(t) \geqslant 4$ by Lemma 3. If $d_{G}(t)=4$, then by applying Lemma 5 to $u t$ we have $d_{G}(w)=5$, a contradiction. Hence, $d_{G}(t)=5$. This means all neighbors of $u$ are of degree 5. By R2.2.2, $c h^{*}(v) \geqslant d_{G}(v)-4-\left(\frac{1}{3}+\frac{4}{\lceil 5 r\rceil-12}\right)-\left(\frac{2}{9}+\frac{4}{3(\lceil 3 r\rceil-6)}\right)-\frac{2}{\lceil 4 r\rceil-9}=\frac{4}{9}-\frac{4}{3(\lceil 3 r\rceil-6)}-\frac{2}{\lceil 4 r\rceil-9}-\frac{4}{\lceil 5 r\rceil-12}$.

If $d_{G}(w)=5$, then $v$ sends charge only to $u$. Hence, $c h^{*}(v) \geqslant d_{G}(v)-4-\left(\frac{1}{3}+\frac{4}{\lceil 5 r\rceil-12}\right)-$ $\left(\frac{1}{3}+\frac{2}{\lceil 3 r\rceil-6}\right)=\frac{1}{3}-\frac{2}{\lceil 3 r\rceil-6}-\frac{4}{\lceil 5 r\rceil-12}$.

It remains to consider the case when $v$ has five $4^{+}$-neighbors. In this case it follows with Claim 15 that $c h^{*}(v) \geqslant d_{G}(v)-4-\left(\frac{1}{3}+\frac{4}{\lceil 5 r\rceil-12}\right)-5\left(\frac{1}{3}\left(\frac{4}{\lceil 5 r\rceil-12}+\frac{2}{\lceil 2 r\rceil-3}\right)\right)=\frac{2}{3}-$ $\frac{32}{3(\lceil 5 r\rceil-12)}-\frac{10}{3(\lceil 2 r\rceil-3)}$.

Since $r>7+\frac{1}{2}$ it follows that $c h^{*}(x) \geqslant 0$ for all $x \in V \cup F$.

The result for $k=6$ in Theorem 2 is implied by the following theorem.

Theorem 16. If $G$ is a planar 6 -critical graph, then $F^{*}(G) \leqslant 3+\frac{2}{5}$.

Proof. Suppose to the contrary that $F^{*}(G)>3+\frac{2}{5}$. Let $\Sigma$ be an embedding of $G$ into the Euclidean plane and $F^{*}(G)=F((G, \Sigma))$. We have

$$
\begin{aligned}
\sum_{f \in F(G)}\left(2 d_{G}(f)-6\right) & =4|E(G)|-6|F(G)| \\
& =4|E(G)|-6(|E(G)|+2-|V(G)|) \quad \text { (by Euler's formula) } \\
& =6|V(G)|-2|E(G)|-12 \\
& \leqslant|V(G)|-15 \quad \text { (by Theorem 9) }
\end{aligned}
$$

and therefore, $-|V(G)|+\sum_{f \in F(G)}\left(2 d_{G}(f)-6\right) \leqslant-15$.

Define the initial charge $\operatorname{ch}(x)$ for each $x \in V(G) \cup F(G)$ as follows: $\operatorname{ch}(v)=-1$ for every $v \in V(G)$ and $\operatorname{ch}(f)=2 d_{G}(f)-6$ for every $f \in F(G)$. It follows from inequality (*) that $\sum_{x \in V(G) \cup F(G)} \operatorname{ch}(x) \leqslant-15$.

A vertex $v$ is called heavy if $d_{G}(v) \in\{5,6\}$ and $v$ is incident with a face of length 4 or 5. A vertex $v$ is called light if $2 \leqslant d_{G}(v) \leqslant 4$ and $v$ is incident with no $6^{+}$-face and with at most one $4^{+}$-face. We say a light vertex $v$ is bad-light if $v$ has a neighbor $u$ such that $d_{G}(u)+d_{G}(v)=8$, and good-light otherwise.

Discharge the elements of $V(G) \cup F(G)$ according to following rules.

R1: every $4^{+}$-face $f$ sends $\frac{2 d_{G}(f)-6}{d_{G}(f)}$ to each incident vertex. 
R2: every heavy vertex sends $\frac{3}{10}$ to each bad-light neighbor, and $\frac{1}{10}$ to each good-light neighbor.

Let $c h^{*}(x)$ denote the final charge of each $x \in V(G) \cup F(G)$ after discharging. On one hand, the sum of charge over all elements of $V(G) \cup F(G)$ is unchanged. Hence, we have $\sum_{x \in V(G) \cup F(G)} c h^{*}(x) \leqslant-15$. On the other hand, we show that $c h^{*}(x) \geqslant 0$ for every $x \in V(G) \cup F(G)$ and hence, this obvious contradiction completes the proof.

It remains to show that $c h^{*}(x) \geqslant 0$ for every $x \in V(G) \cup F(G)$.

Let $f \in F(G)$. If $d_{G}(f)=3$, then no rule is applied for $f$. Thus, $\operatorname{ch}^{*}(f)=\operatorname{ch}(f)=0$.

If $d_{G}(f) \geqslant 4$, then by R1 we have $c h^{*}(f)=\operatorname{ch}(f)-d_{G}(f) \frac{2 d_{G}(f)-6}{d_{G}(f)}=0$.

Let $v \in V(G)$. First we consider the case when $v$ is heavy. On one hand, since $F((G, \Sigma))>3+\frac{2}{5}$, it follows that either $v$ is incident with a $5^{+}$-face and another $4^{+}$-face or $v$ is incident with at least three 4 -faces. In both cases, $v$ receives at least $\frac{13}{10}$ in total from its incident faces by R1. On the other hand, we claim that $v$ sends at most $\frac{3}{10}$ out in total. If $v$ is adjacent to a bad-light vertex $u$, then all other neighbors of $v$ have degree at least 5 by Lemma 5 . Hence, $v$ sends $\frac{3}{10}$ to $u$ by R2 and nothing else to its other neighbors. If $v$ is adjacent to no bad-light vertex, then $v$ has at most three good-light neighbors by Lemma 4 . Hence, $v$ sends $\frac{1}{10}$ to each good-light neighbor by R2 and nothing else to its other neighbors. Therefore, $c h^{*}(v) \geqslant \operatorname{ch}(v)+\frac{13}{10}-\frac{3}{10}=0$.

Second we consider the case when $v$ is not heavy. In this case, $v$ sends no charge out. If $v$ is incident with a $6^{+}$-face, then $v$ receives at least 1 from this $6^{+}$-face by $\mathrm{R} 1$. This gives $c h^{*}(v)=\operatorname{ch}(v)+1=0$. If $v$ is incident with at least two $4^{+}$-faces, then $v$ receives at least $\frac{1}{2}$ from each of them by R1. This gives $\operatorname{ch}^{*}(v)=\operatorname{ch}(v)+\frac{1}{2}+\frac{1}{2}=0$. We are done in both cases above. Hence, we may assume that $v$ is incident with no $6^{+}$-face and with at most one $4^{+}$-face. From $F((G, \Sigma))>3+\frac{2}{5}$ it follows that $v$ is incident to a face $f_{v}$ such that $d_{G}\left(f_{v}\right) \in\{4,5\}$. Since $v$ is not heavy, $2 \leqslant d(v) \leqslant 4$. Hence, $v$ is light by definition. We distinguish two cases by the length of $f_{v}$.

If $d_{G}\left(f_{v}\right)=4$, then by the fact that $F^{*}(G) \geqslant 3+\frac{2}{5}$, we have $d_{G}(v)=2$. By Lemma 3 , both neighbors of $v$ are heavy and $v$ is bad-light. Thus, $v$ receives $\frac{1}{2}$ from $f_{v}$ by $\mathrm{R} 1$ and $\frac{3}{10}$ from each neighbor by R2, yielding $\operatorname{ch}^{*}(v)=\operatorname{ch}(v)+\frac{1}{2}+\frac{3}{10}+\frac{3}{10}>0$.

If $d_{G}\left(f_{v}\right)=5$, then $v$ receives $\frac{4}{5}$ from $f_{v}$. If $v$ is not a bad-light 4-vertex, then Lemma 3 implies that each neighbor of $v$ has degree 5 or 6 . Hence, both of the two neighbors of $v$ contained in $f_{v}$ are heavy. By R2, each of them sends charge at least $\frac{1}{10}$ to $v$, and therefore, $\operatorname{ch}^{*}(v) \geqslant \operatorname{ch}(v)+\frac{4}{5}+\frac{1}{10}+\frac{1}{10}=0$. If $v$ is a bad-light 4-vertex, then Lemma 4 implies that at least one of the two neighbors of $v$ contained in $f_{v}$ is heavy. Thus, this heavy neighbor sends charge $\frac{3}{10}$ to $v$, and therefore, $\operatorname{ch}^{*}(v) \geqslant \operatorname{ch}(v)+\frac{4}{5}+\frac{3}{10}>0$.

\section{Concluding remarks}

Recently, Cranston and Rabern [2] improved Jakobsen's result (Theorem 7) on the lower bound on the number of edges in a 3-critical graph. They gave a computer-aided proof of the following statement.

Theorem 17 ([2]). Every 3-critical graph $G$, other than the Petersen graph with a vertex 
deleted, has $|E(G)| \geqslant \frac{50}{37}|V(G)|$.

Hence, $|E(G)| \geqslant \frac{50}{37}|V(G)|$ for every planar 3-critical graph. By a similar argument as in the proof of Proposition 12, this result improves the bound of $\bar{b}_{3}$ from $6 \leqslant \bar{b}_{3}<8$ to $6 \leqslant \bar{b}_{3}<\frac{100}{13}$. However, the precise values of these parameters are unclear.

Problem 18. What are the precise values of $\bar{b}_{k}$ and $b_{k}^{*}$ ?

By Proposition $12, \bar{F}(G)$ has an upper bound for every critical planar graph $G$. However, this is not always true for class 2 planar graphs. Similarly, Theorems 13 and 16 can not be generalized to class 2 planar graphs.

\section{References}

[1] Y. Bu and W. Wang. Some sufficient conditions for a planar graph of maximum degree six to be Class 1, Discrete Math. 306:1440-1445, 2006.

[2] D. W. Cranston and L. Rabern. Subcubic edge chromatic critical graphs have many edges, arXiv:1506.04225, 2015.

[3] S. Grünewald. Chromatic Index Critical Graphs and Multigraphs, Dissertation, Fakultät für Mathematik, Universität Bielefeld, 2000.

[4] I. T. Jakobsen. On critical graphs with chromatic index 4, Discrete Math. 9:265-276, 1974.

[5] R. Luo, L. Miao, and Y. Zhao. The size of edge chromatic critical graphs with maximum degree 6, J. Graph Theory 60:149-171, 2009.

[6] D. P. Sanders and Y. Zhao. Planar graphs of maximum degree seven are class 1, J. Combin. Theory Ser. B 83:201-212, 2001.

[7] M. Stiebitz, D. Scheide, B. Toft, and L. M. Favrholdt. Edge Graph Coloring: Vizing's Theorem and Goldberg's Conjecture, John Wiley \& Sons, Inc., Hoboken, NJ, 2012.

[8] V. G. Vizing. On an estimate of the chromatic index of a p-graph, Metody Diskret. Analiz 3:25-30, 1964. (in Russian, an English translation can be found in [7])

[9] V. G. Vizing. Critical graphs with given chromatic index, Metody Diskret. Analiz 5:9-17, 1965. (in Russian, an English translation can be found in [7])

[10] W. Wang and Y. Chen. A sufficient condition for a planar graph to be class 1, Theoret. Comput. Sci. 385:71-77, 2007.

[11] Y. Wang and L. Xu. A sufficent condition for a plane graph with maximum degree 6 to be class 1, Discrete Applied Math. 161:307-310, 2013.

[12] D. R. Woodall. The average degree of an edge-chromatic critical graph, Discrete Math. 308:803-819, 2008.

[13] L. Zhang. Every graph with maximum degree 7 is of class 1, Graphs Combin. 16:467-495, 2000.

[14] G. Zhou. A note on graphs of class 1, Discrete Math. 263:339-345, 2003. 